\title{
Clinical Factors Associated With Anxiety and Depression in Korean Women With Abnormal Uterine Bleeding
}

Hae Nam Lee

Catholic University of Korea College of Medicine: Catholic University of Korea School of Medicine Ji Min Seo

Catholic University of Korea College of Medicine: Catholic University of Korea School of Medicine Gi Soo Um

Catholic University of Korea Sungga Hospital: Catholic University of Korea Bucheon Saint Mary's Hospital

Min Jeong Kim ( $\sim$ poouh74@catholic.ac.kr)

The Catholic University of Korea https://orcid.org/0000-0001-9495-2296

Research article

Keywords: Abnormal uterine bleeding, Heavy menstrual bleeding, Anxiety, Depression

Posted Date: October 1st, 2020

DOl: https://doi.org/10.21203/rs.3.rs-74801/v1

License: (c) (i) This work is licensed under a Creative Commons Attribution 4.0 International License. Read Full License 


\section{Abstract}

Background: Abnormal uterine bleeding (AUB) is defined as an abnormality in menstrual bleeding and is common gynecological problem in premenopausal women. Anxiety and depressive disorders were frequently observed in patients with AUB. This study was conducted to investigate the prevalence and the relationship of anxiety and depression in Korean women with AUB.

Methods: The study was a questionnaire study of 124 Korean women aged 15-55 who admitted a single university hospital due to AUB between September 2015 and December 2019. Anxiety, depression were assessed using the Korean Beck Anxiety Inventory (K-BAI), the Korean Beck Depression Inventory-II (K-BDIII). The obstetrical and clinical data were analyzed to assess the association of anxiety and depression with AUB.

Results: Out of 124 patients, $47(37.9 \%)$ met the criteria for anxiety, and 24 (19.5\%) met the criteria for depression. The most common menstrual problems seen were heavy menstrual bleeding (80.7\%), followed by irregular bleeding (62.9\%), dysmenorrhea (55.7\%), and irregular menstrual cycles (33.9\%). More women with AUB in our study had anxiety as measured by the K-BAI and depression as measured by the K-BDI-II. A history of abortion and cesarean section were related with anxiety, whereas a history of minor surgery was related to depression. Anxiety and depression $(r=0.629, p<0.001)$ were correlated with AUB.

Conclusions: Anxiety and depression are underdiagnosed and undertreated in Korean women with AUB symptoms. Screening and appropriate mental health management are needed for women with AUB for women's health.

\section{Background}

Abnormal uterine bleeding (AUB) is defined as an abnormality in the frequency, regularity, amount, or duration of menstrual bleeding and is one of the most common gynecological problems [1, 2]. Estimates of the prevalence of menstrual problems, such as heavy menstrual bleeding (HMB), intermenstrual bleeding, abnormal menstrual cycles, and premenstrual symptoms, range from 19-35\% [3-5]. Menstrual problems are common among premenopausal women, especially just before and during perimenopause. AUB can interfere with the quality of life, and lead women to seek medical care. Although distress is associated with menstrual problems, it is unclear whether mood problems, such as anxiety and depression may increase the susceptibility to menstrual problems. Depression and anxiety are probably the two most common psychological symptoms and they often co-occur. The negative impact of anxietydepression comorbidity is also significant, associated with a more negative course and more negative outcomes [6-8].

Anxiety and depressive disorders were frequently observed in patients with AUB. Although menstrual problems are not life-threatening, they can impose a significant impact on the quality of life of these patients. The identification of these impacts might lead to recognizing the potential risk of psychological 
problems and heighten awareness of mental health and women's health [9]. Understanding the characteristics that predict anxiety and depression may help a clinician identify patients at risk for anxiety or depressive disorders.

To the best of our knowledge, no studies have yet examined the association of anxiety and depression with AUB in Korean women. The purpose of this study was to identify correlations between anxiety and depression in Korean women with AUB. The goals were to identify 1 ) the general characteristics and menstrual problems, 2) the prevalence and risk of anxiety, depression, and 3) the correlations of anxiety and depression with AUB.

\section{Methods}

The study was conducted with 124 consecutive patients who admitted the Department of Obstetrics and Gynecology with complaints of menstrual problems and who were diagnosed according to the FIGO classification system (PALM-COEIN) with AUB between September 2015 and December 2019.

The inclusion criteria were age between 15 and 55 years, premenopausal women and menstrual irregularities continuing for at least three months. The exclusion criteria of the study were the use of psychotropic drugs, such as antidepressants, anxiolytics, or antipsychotics, for any reason in the last six months; a diagnosis of gynecologic cancer; current oral contraceptives or any hormonal therapy, and pregnancy. The flow chart in Fig. 1 indicates the inclusion and exclusion criteria for the patients. This study was obtained from the local ethics committee (The Catholic University of Korea Catholic Medical Center, HC15QISI0078).

The definition of menstrual regularity has changed from one where the shortest to the longest variation was up to 20 days, to a variation of seven to nine days. For practical purposes, this normal variation in cycle length can be alternatively expressed as \pm 4 days. Clinically included is the term HMB, a symptom (not a diagnosis), that has been defined (in clinical situations) by the National Institute for Health and Clinical Excellence as "excessive menstrual blood loss, which interferes with a woman's physical, social, emotional and/or material quality of life" [10-12].

After the survey, the patients were followed- up and treated medically or surgically. Major surgery included total hysterectomy, myomectomy, and ovarian surgery. Minor surgery included endobiopsy, dilatation and curettage, and hysteroscopy.

\section{Measurements}

The Korean Beck Anxiety Inventory (K-BAI) and Korean Beck Depression Inventory-II (K-BDI-II) were used to diagnose anxiety and depression.

The BAl is a 21-item instrument measuring the severity of anxiety symptoms [13]. The total scores range from 0 to 63, with higher scores indicating more severe anxiety symptoms. We used K-BAl that showed excellent internal consistency and good discriminant validity for anxiety disorders [6]. The normal range 
is $0-7$ points (minimal), $8-15$ points is mild, $16-25$ points is moderate, and $26-63$ points is severe. The presence of anxiety was determined based on the K-BAI score, and the subjects were classified into normal subjects (K-BAl score range: $0-15$ ) and subjects with anxiety (K-BAI score range: 16-63).

The BDI was originally developed by Beck in 1961 [14] and revised to BDI-II in 1996 in response to changes in the DSM-IV criteria for the diagnosis of depressive symptoms [15]. The total score ranges from 0 to 63 , with higher scores indicating more severe depressive symptoms. The normal range is $0-3$ points, 4-19 points is mild depression, 20-28 points is moderate, and 29-63 points is severe on the KBDI-II [16]. The presence of depression was determined based on the K-BDI-II score and the subjects were classified as either normal subjects (BDI score range: $0-19$ ) or depressed subjects (BDI score range: 2063) in our study.

\section{Statistical analysis}

All statistical analyses were performed using SAS software ver. 9.4 (SAS Institute Inc., Cary, NC, USA). The Kolmogorov Smirnov test was applied to data that conformed to a normal distribution. For continuous variables that were not normally distributed, the significance of the differences between the groups was tested using the Wilcoxon rank-sum test. The categorical variables were analyzed using the chi-squared test. Pearson's correlation and Spearman's rank correlation were used to analyze the distribution of the anxiety and depression scores. The association between anxiety and depression and clinical data were analyzed by univariable and multivariable logistic regression (anxiety score $\geq 16$, depression score $\geq 20$ ). Multivariable logistic regression was conducted using $p$-values of $<0.05$ in the univariate analysis. For depression, only one variable with statistical significance was not used to perform the multivariable analysis. A $p$-value of 0.05 was considered significant.

\section{Results}

\section{General characteristics of the women}

From September 1, 2015, to December 30, 2019, a total of 131 women with AUB participated in the survey. Seven patients with psychiatric problems confirmed in the past were excluded, and the final research was conducted on 124 women with AUB. Informed consent was obtained from 131 women, the questionnaires were completed, and demographic data and comorbidities were collected from the medical records.

The average age of the study participants was $43.9 \pm 7.9$ years (range, 15-55) and the average body mass index (BMI) was $24.9 \pm 4.4 \mathrm{~kg} / \mathrm{m}^{2}$. Among the participants, 95 women (76.6\%) were married state, and 90 women $(72.6 \%)$ were multiparous.

The most common menstrual problems seen in our study were HMB $(n=100,80.7 \%)$, followed by dysmenorrhea $(n=69,55.7 \%)$ and irregular menstruation $(n=42,33.9 \%$; Table 1$)$. 
Table 1

Comparison of the patients characteristics in the anxiety, depression score

\begin{tabular}{|c|c|c|c|c|c|c|c|}
\hline & $\begin{array}{l}\text { Total } \\
(n= \\
124)\end{array}$ & $\begin{array}{l}\text { Anxiety } \\
\text { score }< \\
16 \\
(n=77)\end{array}$ & $\begin{array}{l}\text { Anxiety } \\
\text { score } \geq \\
16 \\
(n=47)\end{array}$ & $\begin{array}{l}\mathrm{p} \\
\text { value }\end{array}$ & $\begin{array}{l}\text { Depression } \\
\text { score }<20 \\
(n=99)\end{array}$ & $\begin{array}{l}\text { Depression } \\
\text { score } \geq 20 \\
(n=24)\end{array}$ & $\begin{array}{l}p \\
\text { value }\end{array}$ \\
\hline Age (years) & $\begin{array}{l}43.9 \pm \\
7.9\end{array}$ & $\begin{array}{l}43.9 \pm \\
8.0\end{array}$ & $\begin{array}{l}43.9 \pm \\
7.8\end{array}$ & 0.975 & $44.1 \pm 8.0$ & $43.0 \pm 7.3$ & 0.306 \\
\hline $\mathrm{BMI}\left(\mathrm{kg} / \mathrm{m}^{2}\right)$ & $\begin{array}{l}24.9 \pm \\
4.4\end{array}$ & $\begin{array}{l}25.0 \pm \\
4.4\end{array}$ & $\begin{array}{l}24.7 \pm \\
4.4\end{array}$ & 0.637 & $25.0 \pm 4.3$ & $24.5 \pm 4.8$ & 0.431 \\
\hline$<18.5(\%)$ & $4(3.2)$ & $3(75.0)$ & $1(25.0)$ & & $4(100.0)$ & $0(0.0)$ & \\
\hline$\underset{(\%)}{\geq 18.5-<23}$ & $\begin{array}{l}46 \\
(37.1)\end{array}$ & $26(56.5)$ & $20(43.5)$ & & $33(73.3)$ & $12(26.7)$ & \\
\hline$\geq 23-<25(\%)$ & $\begin{array}{l}14 \\
(11.3)\end{array}$ & $8(57.1)$ & $6(42.9)$ & & 12 (85.7) & $2(14.3)$ & \\
\hline$\geq 25$ (\%) & $\begin{array}{l}60 \\
(48.4)\end{array}$ & $40(66.7)$ & $20(33.3)$ & & 50 (83.3) & $10(16.7)$ & \\
\hline $\begin{array}{l}\text { Menarche } \\
\text { (years) }\end{array}$ & $\begin{array}{l}14.3 \pm \\
1.4\end{array}$ & $\begin{array}{l}14.4 \pm \\
1.3\end{array}$ & $\begin{array}{l}14.2 \pm \\
1.5\end{array}$ & 0.566 & $14.3 \pm 1.3$ & $14.2 \pm 1.7$ & 0.821 \\
\hline \multicolumn{8}{|l|}{ Marriage (\%) } \\
\hline no & $\begin{array}{l}23 \\
(18.6)\end{array}$ & $15(65.2)$ & $8(34.8)$ & 0.896 & $18(78.3)$ & $5(21.7)$ & 0.405 \\
\hline marriage & $\begin{array}{l}95 \\
(76.6)\end{array}$ & $58(61.1)$ & $37(38.9)$ & & 77 (81.9) & $17(18.1)$ & \\
\hline Parity & $1.4 \pm 1.0$ & $1.4 \pm 1.1$ & $1.5 \pm 1.0$ & 0.565 & $1.4 \pm 1.0$ & $1.4 \pm 1.1$ & 0.878 \\
\hline 0 & $\begin{array}{l}34 \\
(27.4)\end{array}$ & $23(67.6)$ & $11(32.4)$ & & $28(82.4)$ & $6(17.6)$ & 0.747 \\
\hline$\geq 1$ & $\begin{array}{l}90 \\
(72.6)\end{array}$ & $54(60.0)$ & $36(40.0)$ & 0.434 & $71(79.8)$ & $18(20.2)$ & \\
\hline \multicolumn{8}{|l|}{ Abortion } \\
\hline mean $\pm s d$ & $0.9 \pm 1.3$ & $0.8 \pm 1.4$ & $0.9 \pm 0.9$ & 0.094 & $0.8 \pm 1.2$ & $1.2 \pm 1.3$ & 0.163 \\
\hline \multicolumn{8}{|c|}{ Data are presented as the $\mathrm{n}(\%)$ for categorical variable, unless otherwise indicated. } \\
\hline \multicolumn{8}{|c|}{$p$ value for difference were determined by using chi-square or the wilcoxon rank sum test. } \\
\hline \multicolumn{8}{|c|}{ *statistically significant as $p<0.05$} \\
\hline \multicolumn{8}{|c|}{$\begin{array}{l}\text { BMI, Body mass index; NSD, Normal spontaneous delivery; C/sec, Cesarean section; OC, Oral } \\
\text { contraceptives; AUB, Abnormal uterine bleeding; Hgb, Hemoglobin ; Hct, Hematocrit; DM, Diabetes } \\
\text { mellitus }\end{array}$} \\
\hline
\end{tabular}




\begin{tabular}{|c|c|c|c|c|c|c|c|}
\hline & $\begin{array}{l}\text { Total } \\
(n= \\
124)\end{array}$ & $\begin{array}{l}\text { Anxiety } \\
\text { score < } \\
16 \\
(n=77)\end{array}$ & $\begin{array}{l}\text { Anxiety } \\
\text { score } \geq \\
16 \\
(n=47)\end{array}$ & $\begin{array}{l}\mathrm{p} \\
\text { value }\end{array}$ & $\begin{array}{l}\text { Depression } \\
\text { score }<20 \\
(n=99)\end{array}$ & $\begin{array}{l}\text { Depression } \\
\text { score } \geq 20 \\
(n=24)\end{array}$ & $\begin{array}{l}\mathrm{p} \\
\text { value }\end{array}$ \\
\hline 0 & $\begin{array}{l}65 \\
(52.4)\end{array}$ & $47(72.3)$ & $18(27.7)$ & $0.014^{\star}$ & $54(84.4)$ & $10(15.6)$ & 0.257 \\
\hline$\geq 1$ & $\begin{array}{l}59 \\
(47.6)\end{array}$ & $30(50.8)$ & $29(49.2)$ & & $45(76.3)$ & $14(23.7)$ & \\
\hline \multicolumn{8}{|c|}{ Delivery mode } \\
\hline no & $\begin{array}{l}34 \\
(27.4)\end{array}$ & $23(67.6)$ & $11(32.4)$ & 0.132 & $28(82.4)$ & $6(17.6)$ & 0.373 \\
\hline NSD & $\begin{array}{l}37 \\
(29.8)\end{array}$ & $18(48.6)$ & $19(51.4)$ & & $27(73.0)$ & $10(27.0)$ & \\
\hline $\mathrm{C} / \mathrm{sec}$ & $\begin{array}{l}53 \\
(42.7)\end{array}$ & $36(67.9)$ & $17(32.1)$ & & $44(84.6)$ & $8(15.4)$ & \\
\hline \multicolumn{8}{|c|}{$\begin{array}{l}\text { Menstrual } \\
\text { regularity }\end{array}$} \\
\hline regular & $\begin{array}{l}82 \\
(66.1)\end{array}$ & $47(57.3)$ & $35(42.7)$ & 0.125 & $64(78.0)$ & $18(22.0)$ & 0.196 \\
\hline irregular & $\begin{array}{l}42 \\
(33.9)\end{array}$ & $30(75.0)$ & $12(25.0)$ & & $34(87.2)$ & $5(12.8)$ & \\
\hline \multicolumn{8}{|c|}{ Dysmenorrhea } \\
\hline no & $\begin{array}{l}55 \\
(44.4)\end{array}$ & $33(60.0)$ & $22(40.0)$ & 0.667 & $44(81.5)$ & $10(18.5)$ & 0.806 \\
\hline yes & $\begin{array}{l}69 \\
(55.7)\end{array}$ & $44(63.8)$ & $25(36.2)$ & & $55(79.7)$ & $14(20.3)$ & \\
\hline \multicolumn{8}{|c|}{ Heavy menstrual bleeding } \\
\hline no & $\begin{array}{l}24 \\
(19.4)\end{array}$ & $15(62.5)$ & $9(37.5)$ & 0.964 & $19(79.2)$ & $5(20.8)$ & $\overrightarrow{>} .999$ \\
\hline yes & $\begin{array}{l}100 \\
(80.7)\end{array}$ & $62(62.0)$ & $38(38.0)$ & & $80(80.8)$ & $19(19.2)$ & \\
\hline
\end{tabular}

Data are presented as the $\mathrm{n}(\%)$ for categorical variable, unless otherwise indicated.

$p$ value for difference were determined by using chi-square or the wilcoxon rank sum test.

*statistically significant as $p<0.05$.

BMI, Body mass index; NSD, Normal spontaneous delivery; C/sec, Cesarean section; OC, Oral contraceptives; AUB, Ábnormal uterine bleeding; Hgb, Hemoglobin ; Hct, Hematocrit; DM, Diabetes mellitus 


\begin{tabular}{|c|c|c|c|c|c|c|c|}
\hline & $\begin{array}{l}\text { Total } \\
(n= \\
124)\end{array}$ & $\begin{array}{l}\text { Anxiety } \\
\text { score }< \\
16 \\
(n=77)\end{array}$ & $\begin{array}{l}\text { Anxiety } \\
\text { score } \geq \\
16 \\
(n=47)\end{array}$ & $\begin{array}{l}\mathrm{p} \\
\text { value }\end{array}$ & $\begin{array}{l}\text { Depression } \\
\text { score }<20 \\
(n=99)\end{array}$ & $\begin{array}{l}\text { Depression } \\
\text { score } \geq 20 \\
(n=24)\end{array}$ & $\mathrm{p}_{\text {value }}$ \\
\hline \multicolumn{8}{|c|}{$\begin{array}{l}\text { History of } \\
\mathrm{C} / \mathrm{sec}\end{array}$} \\
\hline no & $\begin{array}{l}70 \\
(56.5)\end{array}$ & $38(54.3)$ & $32(45.7)$ & $0.041^{*}$ & $54(77.1)$ & $16(22.9)$ & 0.282 \\
\hline yes & $\begin{array}{l}54 \\
(43.6)\end{array}$ & $39(72.2)$ & $15(27.8)$ & & $45(84.9)$ & $8(15.1)$ & \\
\hline \multicolumn{8}{|c|}{ History of minor surgery } \\
\hline no & $\begin{array}{l}51 \\
(41.1)\end{array}$ & $32(62.7)$ & 19 (37.3) & 0.901 & $45(90.0)$ & $5(10.0)$ & $0.028^{*}$ \\
\hline yes & $\begin{array}{l}73 \\
(58.9)\end{array}$ & $45(61.6)$ & $28(38.4)$ & & $54(74.0)$ & $19(26.0)$ & \\
\hline \multicolumn{8}{|c|}{ History of OCs } \\
\hline no & $\begin{array}{l}85 \\
(68.6)\end{array}$ & $48(56.5)$ & $37(43.5)$ & 0.057 & $66(78.6)$ & $18(21.4)$ & 0.431 \\
\hline yes & $\begin{array}{l}39 \\
(31.5)\end{array}$ & $29(74.4)$ & $10(25.6)$ & & $33(84.6)$ & $6(15.4)$ & \\
\hline \multicolumn{8}{|c|}{$\begin{array}{l}\text { History of admission due } \\
\text { to AUB }\end{array}$} \\
\hline no & $\begin{array}{l}82 \\
(66.1)\end{array}$ & $49(59.8)$ & $33(40.2)$ & 0.453 & $66(81.5)$ & $15(18.5)$ & 0.699 \\
\hline yes & $\begin{array}{l}42 \\
(33.9)\end{array}$ & $28(66.7)$ & $14(33.3)$ & & $33(78.6)$ & $9(21.4)$ & \\
\hline \multicolumn{8}{|c|}{ History of transfusion } \\
\hline no & $\begin{array}{l}89 \\
(71.8)\end{array}$ & $54(60.7)$ & $35(39.3)$ & 0.603 & $71(80.7)$ & $17(19.3)$ & 0.931 \\
\hline yes & $\begin{array}{l}35 \\
(28.2)\end{array}$ & $23(65.7)$ & $12(34.3)$ & & $28(80.0)$ & $7(20.0)$ & \\
\hline
\end{tabular}

Data are presented as the $\mathrm{n}(\%)$ for categorical variable, unless otherwise indicated.

$p$ value for difference were determined by using chi-square or the wilcoxon rank sum test.

*statistically significant as $p<0.05$.

BMI, Body mass index; NSD, Normal spontaneous delivery; C/sec, Cesarean section; OC, Oral contraceptives; AUB, Abnormal uterine bleeding; Hgb, Hemoglobin ; Hct, Hematocrit; DM, Diabetes mellitus 


\begin{tabular}{|c|c|c|c|c|c|c|c|}
\hline & $\begin{array}{l}\text { Total } \\
(n= \\
124)\end{array}$ & $\begin{array}{l}\text { Anxiety } \\
\text { score }< \\
16 \\
(n=77)\end{array}$ & $\begin{array}{l}\text { Anxiety } \\
\text { score } \geq \\
16 \\
(n=47)\end{array}$ & $\begin{array}{l}\text { palue } \\
\text { valu }\end{array}$ & $\begin{array}{l}\text { Depression } \\
\text { score }<20 \\
(n=99)\end{array}$ & $\begin{array}{l}\text { Depression } \\
\text { score } \geq 20 \\
(n=24)\end{array}$ & $\begin{array}{l}\mathrm{p} \\
\text { value }\end{array}$ \\
\hline \multicolumn{8}{|c|}{$\begin{array}{l}\text { History of iron } \\
\text { supplementation }\end{array}$} \\
\hline no & $\begin{array}{l}45 \\
(36.3)\end{array}$ & $27(60.0)$ & $18(40.0)$ & 0.716 & $34(75.6)$ & $11(24.4)$ & 0.294 \\
\hline yes & $\begin{array}{l}79 \\
(63.7)\end{array}$ & $50(63.3)$ & $29(36.7)$ & & 65 (83.3) & $13(16.7)$ & \\
\hline \multicolumn{8}{|l|}{$\mathrm{Hgb}$} \\
\hline mean $\pm \mathrm{sd}$ & $\begin{array}{l}10.4 \pm \\
2.6\end{array}$ & $\begin{array}{l}10.3 \pm \\
2.6\end{array}$ & $\begin{array}{l}10.7 \pm \\
2.6\end{array}$ & 0.371 & $10.3 \pm 2.5$ & $10.6 \pm 3.0$ & 0.419 \\
\hline $\begin{array}{l}\text { Median } \\
\text { (range) }\end{array}$ & $\begin{array}{l}10.5 \\
(4.0- \\
15.9)\end{array}$ & $\begin{array}{l}10.2(4.0- \\
15.6)\end{array}$ & $\begin{array}{l}10.8 \\
(4.7- \\
15.9)\end{array}$ & & $\begin{array}{l}10.2(5.4- \\
15.9)\end{array}$ & $\begin{array}{l}11.2(4.0- \\
14.5)\end{array}$ & \\
\hline \multicolumn{8}{|l|}{ Hct } \\
\hline mean \pm sd & $\begin{array}{l}32.5 \pm \\
6.6\end{array}$ & $\begin{array}{l}32.1 \pm \\
6.8\end{array}$ & $\begin{array}{l}33.2 \pm \\
6.2\end{array}$ & 0.343 & $32.3 \pm 6.4$ & $33.0 \pm 7.5$ & 0.396 \\
\hline $\begin{array}{l}\text { Median } \\
\text { (range) }\end{array}$ & $\begin{array}{l}32.5 \\
(14.9- \\
47.0)\end{array}$ & $\begin{array}{l}31.9 \\
(14.9- \\
47.0)\end{array}$ & $\begin{array}{l}33.1 \\
(18.7- \\
45.5)\end{array}$ & & $\begin{array}{l}32.0 \\
(18.8- \\
47.0)\end{array}$ & $\begin{array}{l}34.2(14.9- \\
42.9)\end{array}$ & \\
\hline \multicolumn{8}{|c|}{$\begin{array}{l}\text { Medical disorders (DM, Hypertension, } \\
\text { Cancer, Thyroid disorder) }\end{array}$} \\
\hline no & $\begin{array}{l}93 \\
(75.0)\end{array}$ & $56(60.2)$ & 37 (39.8) & 0.454 & $75(80.6)$ & 18 (19.4) & 0.938 \\
\hline yes & $\begin{array}{l}31 \\
(25.0)\end{array}$ & $21(67.7)$ & $10(32.3)$ & & $24(80.0)$ & $6(20.0)$ & \\
\hline \multicolumn{8}{|c|}{ Data are presented as the $n(\%)$ for categorical variable, unless otherwise indicated. } \\
\hline \multicolumn{8}{|c|}{$p$ value for difference were determined by using chi-square or the wilcoxon rank sum test. } \\
\hline \multicolumn{8}{|c|}{ *statistically significant as $p<0.05$} \\
\hline \multicolumn{8}{|c|}{$\begin{array}{l}\text { BMI, Body mass index; NSD, Normal spontaneous delivery; C/sec, Cesarean section; OC, Oral } \\
\text { contraceptives; AUB, Abnormal uterine bleeding; Hgb, Hemoglobin ; Hct, Hematocrit; DM, Diabetes } \\
\text { mellitus }\end{array}$} \\
\hline
\end{tabular}

In the obstetrical and gynecological history, parity, menstrual irregularity, dysmenorrhea, and HMB did not affect the anxiety and depressive state. In the treatment aspect, anxiety and depression were not associated with oral contraceptives use, history of hospitalization due to AUB, transfusion history, or iron 
supplementation. Anxiety and depression scores did not differ according to underlying disease, and hemoglobin and hematocrit levels at the time of the examination (Table 1).

The average number of abortions in the women was $0.9 \pm 1.3$ (range, $0-8$ ), and 65 (52.4\%) women had not had abortions history. In the anxiety group with a K-BAI score of 16 or higher, higher scores were seen in women with AUB and a history of abortion ( $p=0.014$; Table 1$)$.

Among women who delivered, history of Cesarean section (C/sec) was $54(43.6 \%)$ women, and anxiety was higher than in the women without $\mathrm{C} / \mathrm{sec}$ history $(p=0.041)$. There were 73 women $(58.9 \%)$ with AUB and a history of minor surgery and the degree of depression measured by the K-BDI-II was higher than in AUB women who had a history of minor surgery $(p=0.028)$. History of abortion and $\mathrm{C} / \mathrm{sec}$ were associated with anxiety disorder, whereas a history of minor surgery was related to depressive disorder (Table 1).

A total of 124 patients underwent K-BAl evaluation, with a mean score of $14.2 \pm 10.3$ (median 12, range $0-55)$. Forty-seven women (37.9\%) met the criteria for anxiety on the K-BAl evaluation ( $\geq 16)$. A total of 123 patients underwent K-BDI-II evaluations, with an average score of $12.7 \pm 9.0$ (median 11, range 0-47). Twenty-four women (19.5\%) met the criteria for depression on the K-BDI-II ( $\geq 20)$ (Table 2).

Table 2

Distribution of anxiety score (K-BAI) and depression score (K-BDI-II)

\begin{tabular}{|llll|}
\hline \multicolumn{3}{|c|}{ Anxiety score } & Depression score \\
\hline $\mathrm{n}$ & 124 & 123 \\
\hline mean $\pm \mathrm{sd}$ & $14.2 \pm 10.3$ & $12.7 \pm 9.0$ \\
\hline median(range) & $12(0-55)$ & $11(0-47)$ \\
\hline IQR & 6,19 & IQR & 6,16 \\
\hline $0-7$ & $37(29.8)$ & $0-13$ & $75(61.0)$ \\
\hline $08-15$ & $40(32.3)$ & $14-19$ & $24(19.5)$ \\
\hline $16-25$ & $27(21.8)$ & $20-28$ & $16(13.0)$ \\
\hline $26-63$ & $20(16.1)$ & $29-63$ & $8(6.5)$ \\
\hline $\begin{array}{l}\text { K-BAl, Korean-Beck Anxiety } \text { Inventory; K-BDI-II, Korean-Beck Depression Inventory II; IQR, Interquartile } \\
\text { range. }\end{array}$ & & \\
\hline
\end{tabular}

When the relationship between anxiety and depression was analyzed, Pearson's correlation coefficient was $r=0.675(p<0.001)$ and Spearman's rank correlation coefficient was $r=0.629(p<0.001)$, showing a moderately positive correlation (Fig. 2). 
In this study, we performed univariable and multivariable logistic regression of the K-BAI scores, K-BDI-II scores, and clinical and gynecological data (cutoffs: anxiety score $\geq 16$, depression score $\geq 20$ ). Age, $\mathrm{BMI}$, marriage, parity, delivery mode, menstrual regularity, dysmenorrhea, $\mathrm{HMB}$, and history of admission with AUB were not associated with anxiety and depression.

The odds ratio $((\mathrm{OR})=2.48(1.18-5.23), p=0.017)$ for the anxiety scores in univariable logistic regression in the patients with past abortions was high, and the OR was low for anxiety $(\mathrm{OR}=0.47(0.22-0.9), p=$ 0.047 ) in patients with a history of $\mathrm{C} / \mathrm{sec}$. The OR for depression in AUB patients with a history of minor surgery was high $(\mathrm{OR}=2.96(1.05-8.33), p=0.04$, Table 3$)$. 
Table 3

Univariable and multivariable logistic regression (predicted: anxiety score $\geq 16$, depression score $\geq 20$ )

\section{Univariable analysis (predicted : anxiety score $\geq$ 16) \\ Multivariable analysis}

OR $(95 \% \mathrm{Cl})$

$1.00(0.95-1.05) \quad 0.978$

$\begin{array}{ll}\text { adjusted } & \mathrm{p} \\ \mathrm{OR}(95 \% & \text { value }\end{array}$

Univariable analysis (predicted: depression score $\geq 20$ )

OR $(95 \% \mathrm{Cl})$

p

value

Age(years)

BMI $\left(\mathrm{kg} / \mathrm{m}^{2}\right)$

$<18.5$

$0.55(0.06-5.08)$

0.601

$0.30(0.01-$

8.34)

$\geq 18.5-<23$

reference

$0.99(0.30-3.31)$

0.985

0.495

$1.51(0.46-4.94)$

$0.65(0.30-1.44)$

0.294

Menarche(years)

Marriage

no

marriage

reference

$1.17(0.45-3.02)$

0.747

$0.76(0.25-$

2.29)

0.54 (0.11-

2.53)

$0.56(0.22-$

1.42)

$0.93(0.66-$

1.30)

0.468

1.03)

reference

reference

Parity

0

reference

reference

$\geq 1$

$1.37(0.60-3.14)$

0.459

$1.13(0.42-$

3.10)

0.806

Abortion

0 reference

$2.48(1.18-5.23)$

$0.017^{\star}$

2.96

(1.35-

6.49)

reference

reference

$\geq 1$

Delivery mode

*statistically significant as $p<0.05$ in univariate analyses were included in a multivariate analysis.

$p<0.05$ in univariate analyses were included in a multivariate analysis. 


\begin{tabular}{|c|c|c|c|c|c|c|}
\hline & \multicolumn{2}{|c|}{$\begin{array}{l}\text { Univariable analysis } \\
\text { (predicted : anxiety score } \geq \\
\text { 16) }\end{array}$} & \multicolumn{2}{|c|}{$\begin{array}{l}\text { Multivariable } \\
\text { analysis }\end{array}$} & \multicolumn{2}{|c|}{$\begin{array}{l}\text { Univariable analysis } \\
\text { (predicted: depression } \\
\text { score } \geq 20 \text { ) }\end{array}$} \\
\hline no & reference & & & & reference & \\
\hline NSD & $2.15(0.82-5.64)$ & 0.118 & & & $\begin{array}{l}1.67(0.54- \\
5.16)\end{array}$ & 0.369 \\
\hline $\mathrm{C} / \mathrm{sec}$ & $0.98(0.39-2.45)$ & 0.965 & & & $\begin{array}{l}0.84(0.27- \\
2.61)\end{array}$ & 0.76 \\
\hline \multicolumn{7}{|c|}{ Menstrual regularity } \\
\hline regular & reference & & & & reference & \\
\hline irregular & $0.46(0.20-1.06)$ & 0.069 & & & $\begin{array}{l}0.56(0.20- \\
1.59)\end{array}$ & 0.273 \\
\hline \multicolumn{7}{|c|}{ Dysmenorrhea } \\
\hline no & reference & & & & reference & \\
\hline yes & $0.85(0.41-1.77)$ & 0.669 & & & $\begin{array}{l}1.11(0.45- \\
2.71)\end{array}$ & 0.823 \\
\hline \multicolumn{7}{|c|}{ Heavy menstrual bleeding } \\
\hline no & reference & & & & reference & \\
\hline yes & $1.01(0.40-2.52)$ & 0.991 & & & $\begin{array}{l}0.86(0.29- \\
2.55)\end{array}$ & 0.784 \\
\hline \multicolumn{7}{|c|}{ History of $\mathrm{C} / \mathrm{sec}$} \\
\hline no & reference & & reference & & reference & \\
\hline yes & $0.47(0.22-0.99)$ & $0.047^{\star}$ & $\begin{array}{l}0.38 \\
(0.17- \\
0.85)\end{array}$ & $0.018^{*}$ & $\begin{array}{l}0.62(0.25- \\
1.56)\end{array}$ & 0.306 \\
\hline \multicolumn{7}{|c|}{ History of minor surgery } \\
\hline no & reference & & & & reference & \\
\hline yes & $1.04(0.50-2.18)$ & 0.909 & & & $\begin{array}{l}2.96(1.05- \\
8.33)\end{array}$ & $0.04^{*}$ \\
\hline \multicolumn{7}{|c|}{ History of OCs } \\
\hline no & reference & & & & reference & \\
\hline
\end{tabular}

\footnotetext{
*statistically significant as $p<0.05$ in univariate analyses were included in a multivariate analysis. $p<0.05$ in univariate analyses were included in a multivariate analysis.
} 


\begin{tabular}{|c|c|c|c|c|c|}
\hline \multirow[b]{2}{*}{ yes } & \multicolumn{2}{|c|}{$\begin{array}{l}\text { Univariable analysis } \\
\text { (predicted : anxiety score } \geq \\
\text { 16) }\end{array}$} & \multirow[t]{2}{*}{$\begin{array}{l}\text { Multivariable } \\
\text { analysis }\end{array}$} & \multicolumn{2}{|c|}{$\begin{array}{l}\text { Univariable analysis } \\
\text { (predicted: depression } \\
\text { score } \geq 20 \text { ) }\end{array}$} \\
\hline & $0.46(0.20-1.06)$ & 0.068 & & $\begin{array}{l}0.70(0.26- \\
1.89)\end{array}$ & 0.478 \\
\hline \multicolumn{6}{|c|}{ History of admission due to AUB } \\
\hline no & \multicolumn{3}{|l|}{ reference } & \multicolumn{2}{|l|}{ reference } \\
\hline yes & $0.75(0.35-1.64)$ & 0.472 & & $\begin{array}{l}1.22(0.49- \\
3.04)\end{array}$ & 0.675 \\
\hline \multicolumn{6}{|c|}{ History of transfusion } \\
\hline no & \multicolumn{3}{|l|}{ reference } & \multicolumn{2}{|l|}{ reference } \\
\hline yes, & $0.82(0.36-1.85)$ & 0.626 & & $\begin{array}{l}1.08(0.41- \\
2.84)\end{array}$ & 0.884 \\
\hline \multicolumn{6}{|c|}{ History of iron supplementation } \\
\hline no & \multicolumn{3}{|l|}{ reference } & \multicolumn{2}{|l|}{ reference } \\
\hline yes & $0.87(0.41-1.84)$ & 0.713 & & $\begin{array}{l}0.62(0.25- \\
1.52)\end{array}$ & 0.293 \\
\hline $\mathrm{Hgb}$ & $1.07(0.92-1.23)$ & 0.383 & & $\begin{array}{l}1.04(0.87- \\
1.24)\end{array}$ & 0.662 \\
\hline Hct & $1.03(0.97-1.09)$ & 0.374 & & $\begin{array}{l}1.02(0.95- \\
1.09)\end{array}$ & 0.646 \\
\hline \multicolumn{6}{|c|}{ Medical disorders (DM, HTN, Cancer, Thyroid disorder) } \\
\hline no & \multicolumn{3}{|l|}{ reference } & \multicolumn{2}{|l|}{ reference } \\
\hline yes & $0.74(0.31-1.73)$ & 0.483 & & $\begin{array}{l}1.08(0.39- \\
2.99)\end{array}$ & 0.878 \\
\hline \multicolumn{6}{|c|}{ *statistically significant as $p<0.05$ in univariate analyses were included in a multivariate analysis. } \\
\hline \multicolumn{6}{|c|}{$p<0.05$ in univariate analyses were included in a multivariate analysis. } \\
\hline
\end{tabular}

Among the patients, 60 women (48.4\%) had undergone hysterectomy, five women had undergone myomectomy, 45 women had undergone hysteroscopy or endobiopsy, and 16 women used the intrauterine device (Mirena). After surgery, the pathologic findings revealed that 76 women (61.3\%) had uterine disorders (leiomyoma and adenomyosis), 34 (27.4\%) women had endometrial disorders (endometrial hyperplasia and endometrial polyps), and three (2.4\%) women had endometrial malignancies (atypical endometrial hyperplasia and endometrial cancer).

\section{Discussion}


The worldwide impact of AUB in the reproductive years is noteworthy, with a prevalence of approximately $3-30 \%$ among reproductive-aged women. Many of the published studies are restricted to estimates of the prevalence of the symptoms of HMB. When other symptoms, particularly those of irregular and intermenstrual bleeding are included, the prevalence rises to $35 \%$ or higher [17]. AUB not only gives discomfort and anxiety to women, but it can be accompanied by depression when there is persistent AUB. In AUB patients who visit the hospital with anxiety and depression, proper management is necessary, but the importance of psychological and risk evaluations has been underestimated.

Menstrual-related problems are associated with substantial psychological distress, a finding that confirms results reported in clinical cases and strongly supports the claim that menstrual-related problems pose important public health implications $[18,19]$. Mood and anxiety disorders, particularly major depression (15.6\%), generalized anxiety disorder (18.8\%), and obsessive-compulsive disorder (22.9\%) were frequently observed in patients with AUB [1].

Strine et al. [4] suggested that menstrual-related problems in women pose considerable public health implications as they were reported by nearly $19 \%$ of U.S. women. Additionally, those with menstrualrelated problems are between 1.7 and 3.0 times more likely to report insomnia, sleepiness, recurrent pain, sadness, nervousness, restlessness, hopelessness, and worthlessness.

The lifetime prevalence of mental illness in Koreans is known to be $25.4 \%$. According to an epidemiological survey of mental disorders in Korea conducted by the Ministry of Health and Welfare, the estimated lifetime prevalence of anxiety disorders for Korean adults was 9.3\% (male 6.7\%, female 11.7\%) and the 1-year prevalence of anxiety disorders in Korean adults was $5.7 \%$ (male 3.8\%, female 7.5\%) [20]. The prevalence of major depression is quite wide and ranges between 8.2 and $67 \%$. According to the level of mental health among Koreans, the rate of experience of depression was $13 \%$, and the prevalence of depression was $5.0 \%[1,20]$.

More women with AUB in our study had anxiety as measured by the K-BAI ( $7.5 \%$ in the general population and $37.9 \%$ in this study) and depression as measured by the K-BDI-II ( $5 \%$ in the general population and $19.5 \%$ in this study). Our study found that the prevalence of anxiety and depression was higher in AUB patients.

Mood and anxiety disorders associated with irregular menstruation may also be associated with different etiologies. It is well-documented that depression is seen more frequently in women during premenstrual, postnatal, and menopausal periods due to the fluctuations in hormonal levels during these periods [21]. However, it is difficult to clearly confirm the relationship between psychological aspects and physical symptoms.

When all factors are taken into consideration, a bidirectional relationship between AUB and psychiatric disorders may be observed. Kayhan et al. [1] reported that psychiatric disorders play a more important role than AUB because the latter frequently occurs together with stressful events and psychiatric disorders, but once these events or disorders are resolved, the menstrual cycle becomes regular again. 
Although the relationship between AUB and anxiety, depression is difficult to know clearly, research on the association has important implications for women's health.

We hypothesized that age, BMI, obesity, abortion history, surgery-related delivery or gynecologic problems, menstruation cycles, dysmenorrhea, anemia, and medical disorders may be associated with anxiety and depression in the presence of AUB, but there was no clear relationship in our study.

In this study, anxiety and depression showed a moderately positive correlation with AUB, indicating that it is necessary to closely monitor and manage whether anxiety or depression accompany women with AUB.

Women with past mood disorders were more likely to report heavy bleeding symptoms, independent of known risk factors for heavy bleeding, such as high BMI, fibroids, early perimenopause, and mood disorders, occurring simultaneously with heavy bleeding. Mood disorder has been shown to be a risk factor for the subsequent development of important health disorders, such as diabetes, cardiovascular disease, pain, backache, and dizziness [3, 22].

In the case of AUB, anxiety increased with a history of abortion, and anxiety scores were low in women with a history of $\mathrm{C} / \mathrm{sec}$. Although it is difficult to know the relationship clearly, it seems that anxiety increases when a loss is experienced, such as abortion, and sensitivity to anxiety decreases when a major operation such as $\mathrm{C} / \mathrm{sec}$ has already been performed. However, it seems that parity, irregular menstruation, and dysmenorrhea did not significantly affect anxiety, especially in the presence of AUB in our study.

The degree of depression was increased in women with a history of minor surgery, which seemed to be because it affected patient mood in the presence of AUB. However, minor surgery did not appear to affect anxiety.

There were several limitations to our study. We were unable to determine if psychological distress and adverse health behaviors were related to AUB, and unable to exactly identify where during the menstrual cycle the psychological and behavioral associations were more evident. The K-BAI and K-BDI-II were not originally developed as diagnostic tools. Our study could not conclude a causal relationship between menstrual-related problems, emotional well-being, and psychological problems.

The women who participated in the survey are likely to have sampling bias error because AUB symptoms interfere with daily life and all participated women were admitted hospital due to management of AUB. Due to a lack of other similar studies in the literature, an analysis of the difficulties and limitations of the current study in comparison to other studies was not possible.

\section{Conclusions}

In Korean women, AUB seems to increase the risk of anxiety and depression. Although anxiety and depression were assessed through the K-BAl, K-BDI-II, respectively, this is a meaningful study by suggesting that anxiety and depression can be severe in women with AUB. Thus, including an 
assessment of AUB as part of the standard evaluation of women may better enable healthcare providers to recognize and treat potential manifestations of these symptoms.

\section{Abbreviations}

AUB

abnormal uterine bleeding; K-BAl:Korean Beck Anxiety Inventory; K-BDI-II:Korean Beck Depression Inventory-ll; C/sec:cesarean section; HMB:heavy menstrual bleeding

\section{Declarations}

\section{Acknowledgements}

Not applicable.

\section{Author's contributions}

HNL, MJK-designed the study concept and wrote the manuscript. JMS-interpreted data, statistical analysis of the results. GSU, MJK contributed to data analysis. All authors read and approved the final manuscript.

\section{Funding}

The statistical consultation (interpretation of data) was supported by a grant ( $\mathrm{HC14C1062)}$ from the Korea Health Technology R\&D Project through the Korean Health Industry Development Institute (KHIDI) funded by the Ministry of Health \& Welfare, Republic of Korea.

\section{Availability of data and materials}

The datasets used during the current study can also be obtained the corresponding author on reasonable request.

\section{Ethics approval and consent to participate}

This study was approved by the Ethics Committee of the Catholic University of Korea (approval number: HC15QISI0078). This study was retrospective clinical study using medical record, written informed consent was waivered by the ethics committee.

\section{Consent for publication}

Not applicable.

\section{Competing interests}

The authors declare that they have no competing interests. 


\section{References}

1. Kayhan F, Alptekin H, Kayhan A. Mood and anxiety disorders in patients with abnormal uterine bleeding. Eur J Obstet Gynecol Reprod Biol. 2016;199:192-7.

2. Spencer CP, Whitehead MI. Endometrial assessment re-visited. Br J Obstet Gynaecol. 1999;106(7):623-32.

3. Bromberger JT, Schott LL, Matthews KA, Kravitz HM, Randolph JF, Jr., Harlow S, et al. Association of past and recent major depression and menstrual characteristics in midlife: Study of Women's Health Across the Nation. Menopause. 2012;19(9):959-66.

4. Strine TW, Chapman DP, Ahluwalia IB. Menstrual-related problems and psychological distress among women in the United States. J Womens Health (Larchmt). 2005;14(4):316-23.

5. Hurskainen R, Aalto AM, Teperi J, Grenman S, Kivelä A, Kujansuu E, et al. Psychosocial and other characteristics of women complaining of menorrhagia, with and without actual increased menstrual blood loss. BJOG. 2001;108(3):281-5.

6. Lee K, Kim D, Cho Y. Exploratory factor analysis of the Beck Anxiety Inventory and the Beck Depression Inventory-II in a psychiatric outpatient population. J Korean Med Sci. 2018;33(16):e128.

7. Kessler RC, Chiu WT, Demler O, Merikangas KR, Walters EE. Prevalence, severity, and comorbidity of 12-month DSM-IV disorders in the National Comorbidity Survey Replication. Arch Gen Psychiatry. 2005;62(6):617-27.

8. Lamers F, van Oppen P, Comijs HC, Smit JH, Spinhoven P, van Balkom AJ, et al. Comorbidity patterns of anxiety and depressive disorders in a large cohort study: the Netherlands Study of Depression and Anxiety (NESDA). J Clin Psychiatry. 2011;72(3):341-8.

9. Nur Azurah AG, Sanci L, Moore E, Grover S. The quality of life of adolescents with menstrual problems. J Pediatr Adolesc Gynecol. 2013;26(2):102-8.

10. Munro MG, Critchley HOD, Fraser IS. The two FIGO systems for normal and abnormal uterine bleeding symptoms and classification of causes of abnormal uterine bleeding in the reproductive years: 2018 revisions. Int J Gynaecol Obstet. 2018;143(3):393-408.

11. Harlow SD, Lin X, Ho MJ. Analysis of menstrual diary data across the reproductive life span applicability of the bipartite model approach and the importance of within-woman variance. J Clin Epidemiol. 2000;53(7):722-33.

12. National Institute for Health and Care Excellence. Heavy menstrual bleeding: assessment and management [Internet]. London: National Institute for Health and Care Excellence; 2018 [updated 2020 Mar 31]. Available from: https://www.nice.org.uk/guidance/

13. Beck AT, Epstein N, Brown G, Steer RA. An inventory for measuring clinical anxiety: psychometric properties. J Consult Clin Psychol. 1988;56(6):893-7.

14. Beck AT, Ward CH, Mendelson M, Mock J, Erbaugh J. An inventory for measuring depression. Arch Gen Psychiatry. 1961;4:561-71. 
15. Beck AT, Steer RA, Brown GK. BDI-II : Beck depression inventory. San Antonio: Psychological Corp.; 1996.

16. Jo SA, Park MH, Jo I, Ryu SH, Han C. Usefulness of Beck Depression Inventory (BDI) in the Korean elderly population. Int J Geriatr Psychiatry. 2007;22(3):218-23.

17. Kazemijaliseh H, Ramezani Tehrani F, Behboudi-Gandevani S, Khalili D, Hosseinpanah F, Azizi F. A population-based study of the prevalence of abnormal uterine bleeding and its related factors among Iranian reproductive-age women: an updated data. Arch Iran Med. 2017;20(9):558-63.

18. Halbreich U, Borenstein J, Pearlstein T, Kahn LS. The prevalence, impairment, impact, and burden of premenstrual dysphoric disorder (PMS/PMDD). Psychoneuroendocrinology. 2003;28 Suppl 3:1-23.

19. Sternfeld B, Swindle R, Chawla A, Long S, Kennedy S. Severity of premenstrual symptoms in a health maintenance organization population. Obstet Gynecol. 2002;99(6):1014-24.

20. Ministry of Health and Welfare. Mental disease survey [Internet]. Seoul: Statistics Korea; 2016 [cited 2018 Nov 27]. Available from: https://meta.narastat.kr/metasvc/index.do?confmNo=117050

21. Studd JW. A guide to the treatment of depression in women by estrogens. Climacteric. 2011;14(6):637-42.

22. Hotopf M, Mayou R, Wadsworth M, Wessely S. Temporal relationships between physical symptoms and psychiatric disorder. Results from a national birth cohort. Br J Psychiatry. 1998;173:255-61.

\section{Figures}




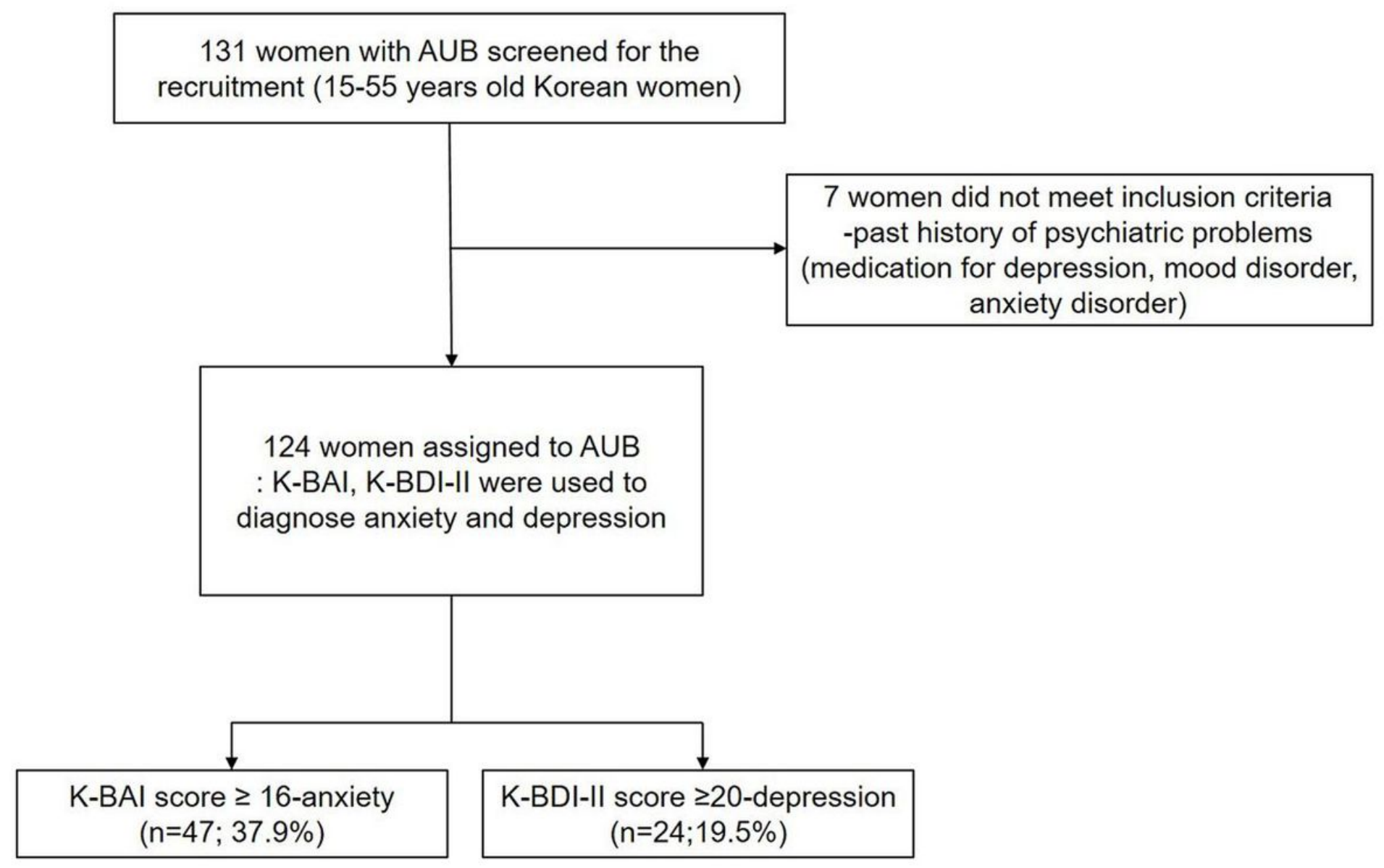

\section{Figure 1}

Flow chart of the participants through the trial. AUB, Abnormal uterine bleeding; K-BAl, Korean Beck Anxiety Inventory; K-BDI-II, Korean Beck Depression Inventory-II 


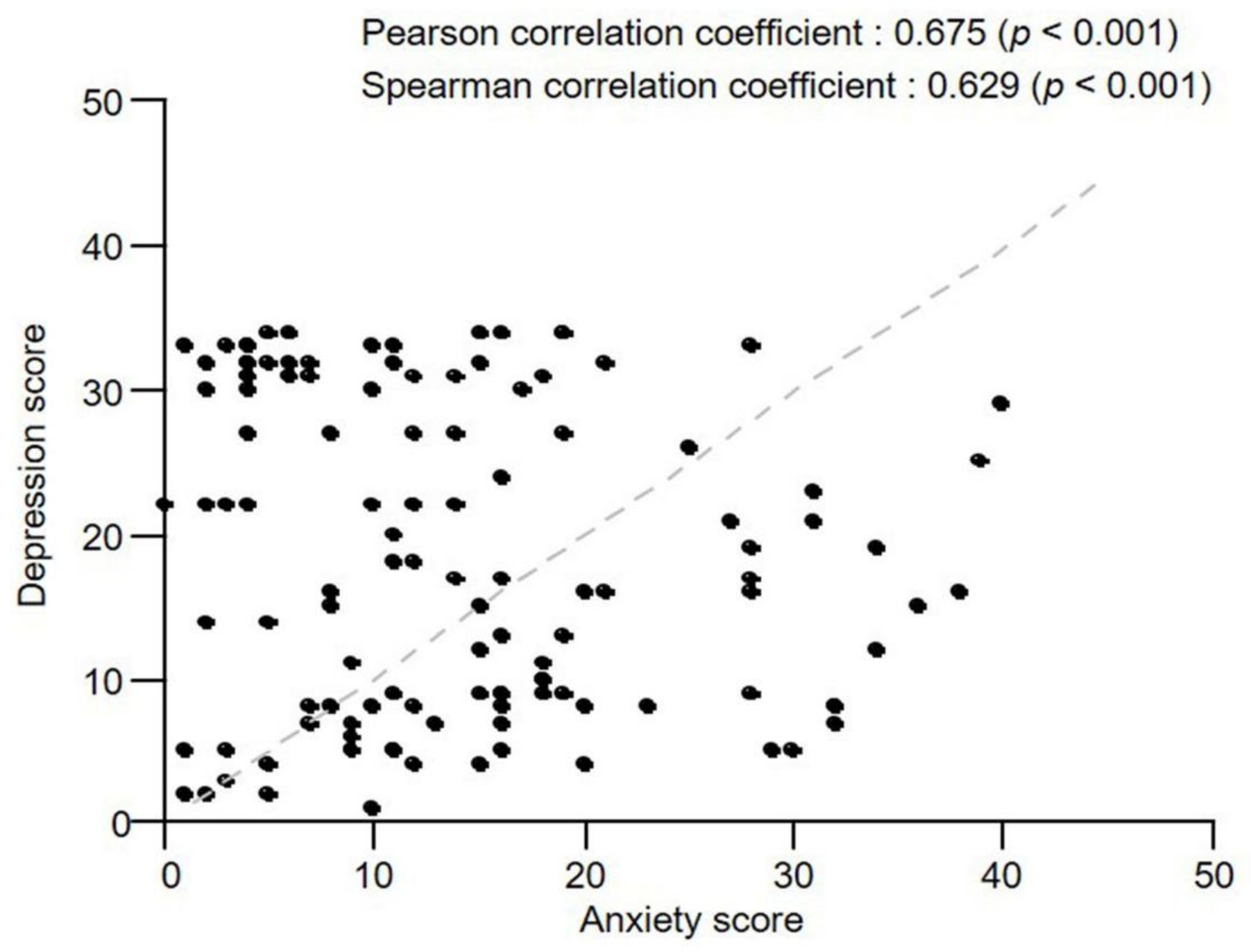

Figure 2

Correlation coefficients for anxiety with depression. 